\title{
Evaluation of the In Vivo Biocompatibility of Hydroxyapatite Granules Incorporated with Zinc Ions
}

\author{
Ingrid Russoni de Lima ${ }^{\mathrm{a}, \mathrm{b}, \mathrm{e}}$, Gutemberg Gomes Alves ${ }^{\mathrm{c}, \mathrm{d}}$, Gustavo Vicentis de Oliveira Fernandes ${ }^{\mathrm{c}}$ \\ Eliane Pedra Dias ${ }^{\mathrm{e}}$, Glória de Almeida Soares ${ }^{\mathrm{a}}$,José Mauro Granjeiro ${ }^{\mathrm{c}, \mathrm{d}, *}$ \\ aPrograma de Engenharia Metalúrgica e de Materiais, \\ Universidade Federal do Rio de Janeiro - UFRJ, Rio de Janeiro, RJ, Brazil \\ ${ }^{\mathrm{b}}$ Departamento de Formação Específica, Faculdade de Odontologia, \\ Universidade Federal Fluminense - UFF, Pólo Universitário, Nova Friburgo, RJ, Brazil \\ ${ }^{\mathrm{C} D e p a r t a m e n t o}$ de Biologia Celular e Molecular, Instituto de Biologia, \\ Universidade Federal Fluminense - UFF, Niterói, RJ, Brazil \\ ${ }^{\mathrm{d}}$ Unidade de Pesquisa Clínica, Hospital Universitário Antonio Pedro - HUAP, \\ Universidade Federal Fluminense - UFF, Niterói, RJ, Brazil \\ ePós-graduação em Patologia, Hospital Universitário Antonio Pedro - HUAP, \\ Universidade Federal Fluminense - UFF, Niterói, RJ, Brazil
}

Received: October 15, 2010; Revised: December 13, 2010

\begin{abstract}
The incorporation of zinc onto biomaterials may improve their biological properties, since it is an essential trace element, involved on important steps of bone remodeling. The aim of this work was to evaluate the in vivo biocompatibility of previously characterized hydroxyapatite granules incorporated with $5 \%$ zinc (ZnHA), through a subcutaneous grafting test. Granules produced from powders of ZnHA or stoichiometric HA were subcutaneously grafted onto Balb/c mice, and histomorphometrical analysis was performed at 7, 14, 21, 28 or 60 days after grafting. HA and ZnHA induced a similar foreign body reaction, with formation of granulome and the presence of a fibrous capsule. Granulocytes were virtually absent, and agranulocytes decreased with time. ZnHA treated samples presented faster reduction on multinucleated giant cells and increase on observed angiogenesis, as compared to HA. It could be concluded that ZnHA granules are biocompatible. Further study should better address its potential use for bone therapy.
\end{abstract}

Keywords: hydroxyapatite, zinc, biocompatibility, histomorphometry

\section{Introduction}

A recurrent issue on bone therapy and regenerative medicine is the search for new materials which are able to improve bone regeneration, thus reducing the consequences of bone loss on events such as trauma, fractures or teeth extraction. Among several successful biomaterials, it is possible to highlight the role of synthetic bioactive ceramics, which represent very desirable characteristics such as low immunogenicity, no risks of transmitting diseases from donors, as well as the availability for unlimited mass production ${ }^{1}$. Among such materials, it could be highlight the employment of calcium phosphates, especially hydroxyapatite (HA), which presents several chemical, biological and crystallographic similarities with the mineral phase of human bone, therefore being recognized as very biocompatible, and presenting well known osteoconductive properties ${ }^{2,3}$.

Another important characteristic of HA is the possibility of incorporation of other components, such as metallic ions, on its structure $^{4-6}$, thus promoting alterations on important physicochemical properties with impact on the biological response to this biomaterial. Also, this substitution may include bioactive elements or ions, which could also modulate important events related to bone regeneration, as they are released from HA onto biological media ${ }^{7}$. In this context, Zinc ions may represent strong candidates for incorporation on bioactive materials. Zinc is an essential trace element, which is involved on the regulation of osteoblast and osteoclast activities, the production of collagen matrix and expression of Sodium-Dependent Vitamin C Transporter 2 (SVCT 2), involved on osteoclast differentiation ${ }^{8}$. Zinc is also a co-factor for alkaline phosphatase, an important enzyme on bone mineralization'. Furthermore, there is clinical evidence of a relationship between Zinc deficiency and osteoporosis ${ }^{10}$.

Different studies in vivo and in vitro have shown good results with the incorporation of $\mathrm{Zn}$ on several biomaterials, such as glass ceramics and $\mathrm{TCP}^{11-15}$. In such cases, the addition of Zinc has stimulated bone growth and mineralization, through its direct action on osteoblasts and inhibition of osteoclasts ${ }^{12,16}$. Regarding hydroxyapatite, previous work has shown that zinc ions may be incorporated on the structure of HA granules during synthesis, thus obtaining a bioceramic material (5\% mol ZnHA) of small crystal size, and able to release considerable amounts of free zinc ions on media during degradation tests ${ }^{14}$. On the other hand, the literature is very limited concerning the impact of Zinc incorporation on the in vivo biocompatibility of hydroxyapatite-based granules ${ }^{11,13}$. In this work, it was shown that $\mathrm{ZnHA}$ was able to perform very similarly to stoichiometric HA granules on their biological responses after being grafted subcutaneously on Balb/c 3T3 mice. 


\section{Materials and Methods}

\subsection{Synthesis of hydroxyapatite and zinc-hydroxyapatite powders}

Hydroxyapatite powders were produced on CBPF (Brazilian Center for Physics Research) by the co-precipitation method, employing calcium nitrate and ammonium phosphate solutions in the presence or absence of zinc at a theoretical molar ratio of $5 \%$, considering the ratio for the content of $\mathrm{Ca}, \mathrm{P}$ and $\mathrm{Zn}$ as $9.5 \mathrm{Ca} / 0.5 \mathrm{Zn} / 6 \mathrm{P}$, described previously by Costa et al. ${ }^{17}$.

\subsection{Preparation of granules}

Granules were obtained from the previously described powders, employing stearic acid on a granulometric range between $0.5-1.5 \mathrm{~mm}$ as a porogeneic agent, in the proportion (wt. (\%)) of 60/40. Samples with $1 \mathrm{~g}$ were taken to an uniaxial press $(27.8 \mathrm{MPa})$ to produce $12 \mathrm{~mm}$ diameter discs. The dense discs thus obtained were calcinated at $1200^{\circ} \mathrm{C}$, obtaining porous discs free of stearic acid particles (through volatilization). The porous discs were grinded and sieved to a size of 250 to $1000 \mu \mathrm{m}$. The physicochemical characterization of the granules thus obtained was described previously ${ }^{14}$. Before grafting, the materials were sterilized though gamma radiation.

\subsection{In vivo biocompatibility test}

\subsubsection{Experimental groups}

Fifty Balb/c mice were randomly distributed on 2 experimental groups ( $\mathrm{n}=25 \mathrm{each}$ ), namely HA and ZnHA. Animals from each group were grafted subcutaneously with their respective materials (HA or ZnHA), and subdivided on groups of 5 individuals to be sacrificed at different experimental times $(7,14,21,28$ or 60 days after grafting). The rational for analysis of new biomaterials implies the cytotoxicity evaluations ${ }^{14}$, followed by the determination of subcutaneous response and intra-bony biocompatibility, as preconized by ISO 10993-5 and -6, former one applied in the present study.

\subsubsection{Surgical procedures}

The surgical steps were initiated with general anesthesia (trough the association of 3:1 ketamine and xylazine chloridrates), followed by trichotomy and antisepsis of the dorsal region. Access to the subcutaneous tissue was obtained by $2 \mathrm{~cm}$ incisions, allowing the grafting of a total amount of $50 \mathrm{mg}$ granules of the either $\mathrm{ZnHA}$ or stoichiometric HA. After suture, animals were maintained during all the experimental periods with ration and water ad libitum, at room temperature and a 12 hours light/dark cycle. By the end of each experimental time, animals were sacrificed and samples were collected from the grafted areas (following animal welfare standards). Samples, represented by the granulome (characteristic of a foreign body reaction) plus a safety border of around $0.5 \mathrm{~cm}$, were then fixed on $10 \%$ formaldehyde buffered solution for 48 hours, followed by washing on running water and demineralization with EDTA. The demineralization process was monitored by radiography on a weekly basis, until radiolucidity was attained. Samples were then dehydrated in ethanol, diaphanized in xylol and finally impregnated and embedded in paraffin. Sections were cut at 5-6 $\mu \mathrm{m}$ thickness, and stained with Hematoxylin and Eosin (HE).

\subsubsection{Histomorphometrical analysis}

Subjective histological evaluations were performed in order to detect cell events involved on the tissue response to both materials (HA and 5\% mol ZnHA), describing: i) the type of inflammatory infiltrate (chronic or acute); ii) the presence of granulocytes, agranulocytes and multinucleated giant cells; iii) fibrous tissue deposition; iv) angiogenesis; and v) the presence of necrotic areas. For the morphometric analysis, slides were observed on a bright-field microscope (Jenaval Universal, Carl Zeiss-Jena), with an 80A filter (color temperature correction). Fifteen randomly selected and non-overlapping fields were colleted for each slide, with a digital camera (Sony Cybershot DSC-W300; 13.6 megapixels) and analyzed with the help of the software Image Pro Plus 6.0.

\subsection{Statistical analysis}

The results of all experiments were analyzed with the software InStat $^{\circledR}$ (GraphPad Software Inc, USA). Means and SD were evaluated for normality, and submitted to either one-way ANOVA with a Bonferroni or Tukey post-test, or a Kruskal-Wallis non-parametric test with Dunnet post-test. The alpha error was set as 0.05 .

\section{Results and Discussion}

In vivo studies with grafted materials, together with in vitro cytotoxicity assays, are very relevant tools available in materials research, as they help to predict the general biological response to material surfaces prior to the clinical application of novel biomaterials. In this regard, in vivo tests employing the use of subcutaneous grafting are suggested as an interesting approach by different international standards for material evaluation ${ }^{18,19}$. It has led us to choose such an in vivo model to access the biocompatibility of ZnHA granules.

Several samples were obtained from excision of subcutaneous grafted areas at different experimental times. During the process of demineralization of the samples on EDTA, as followed through radiographic analysis, it could be observed that samples obtained from animals grafted with ZnHA lost their radiopacity on lower times (from 15 to 35 days) than those obtained from the stoichiometric HA group (from 61 to 71 days).

A qualitative microscopic analysis from these samples revealed focal regions with a strong presence of vesicle-rich, macrophage-like cells, on both experimental groups (ZnHA and HA) at day 7 after grafting. It was observed an intense inflammatory infiltrate, rich on agranulocytes and inflammatory multinucleated giant cells (MnGCs), circumscribing the grafted granules (Figure 1). Granulocytes, if present, were very sparsely distributed. The inflammatory process was organized as a foreign body granulome, peripherically surrounded by a discrete layer of fibrous tissue, which seemed more thick on HA group, as compared to ZnHA (Figure 1a). On the other hand, the presence of blood vessels was more evidenced on ZnHA samples (Figure 1b).

At day 14, the presence of unmodelled connective tissue became evident on stoichiometric HA treated samples (Figure 2a). For the ZnHA samples, however, an intense inflammatory infiltrate still persisted, rich on agranulocytes and MnGCs, and presenting intense angiogenesis (Figure 2b, c).

Between days 21 and 28, fibroblasts were predominant and disposed on a more organized manner, presenting areas of dense conjunctive tissue on both experimental groups (Figure 3 ). The inflammatory infiltrate also remained rich on agranulocytes on both groups, but an intense presence of vascularization was more evident on ZnHA group. By the end of 60 days, dense connective tissue was predominant on both groups, with proximal regions of loose connective tissue, and the presence of congested, large caliber blood vessels (Figure 4).

The results obtained with cell distribution by histomorphometrical analysis of the grafted areas presented on Figure 5. It is possible to observe that the estimated number of agranulocytes have changed 


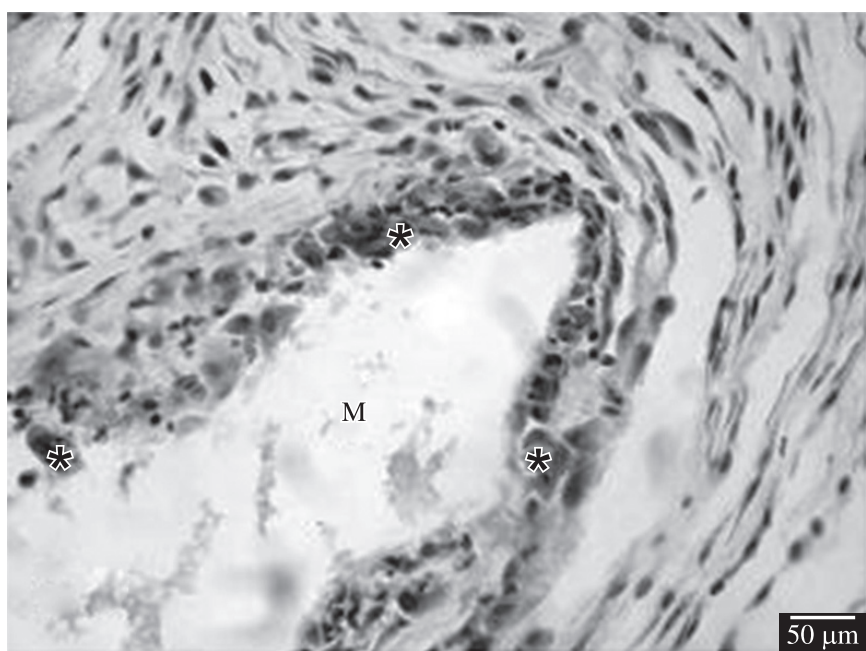

(a)

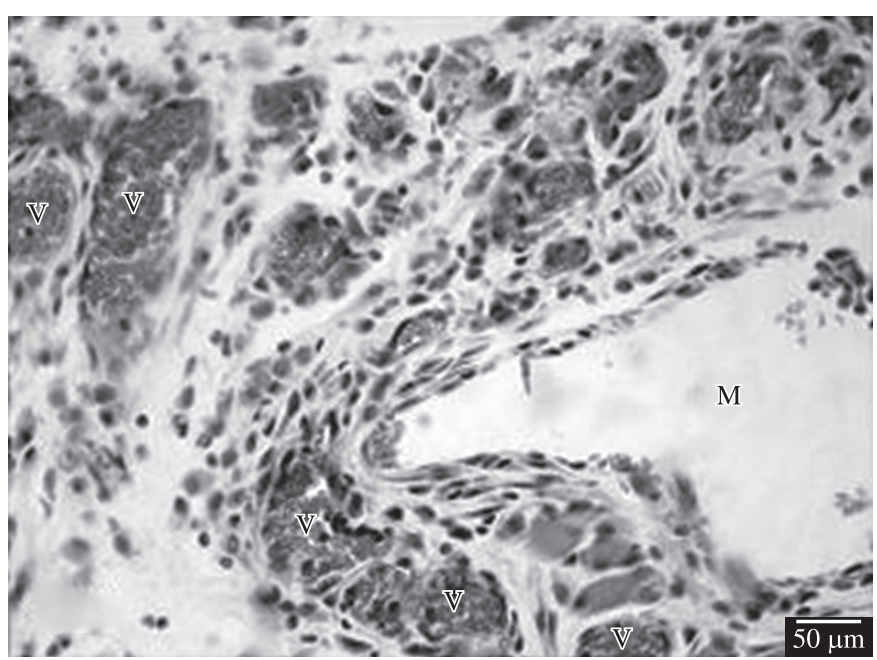

(b)

Figure 1. Histological sections, stained with HE, 7 days after grafting with HA (a) or ZnHA (b). Asterisks indicate Multinucleated Giant Cells. (M) indicates the grafted area. (V) indicates blood vessels.

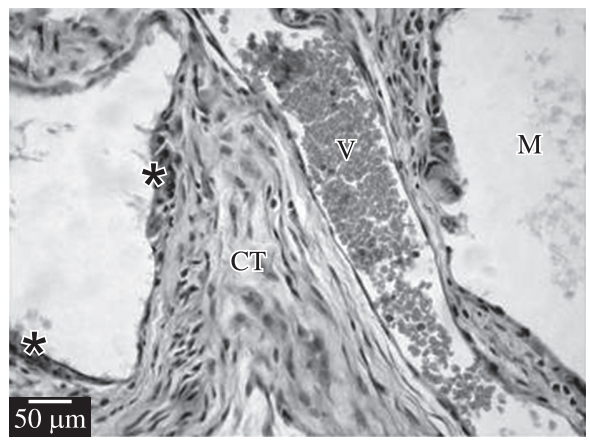

(a)

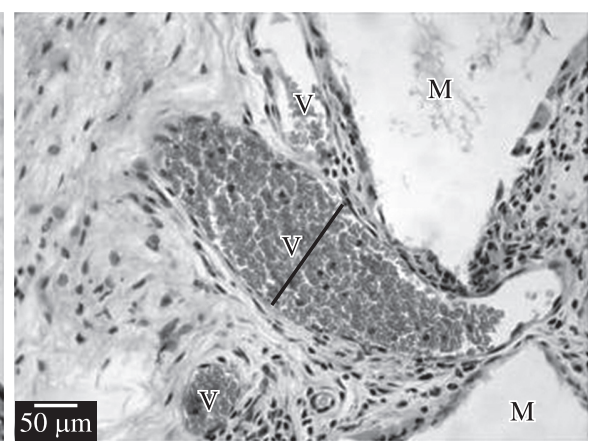

(b)

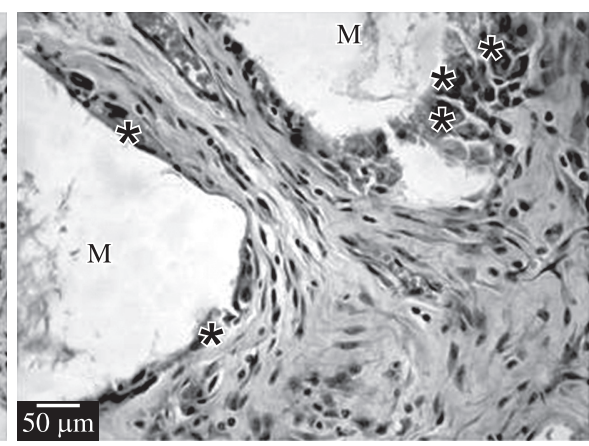

(c)

Figure 2. Histological sections, stained with HE, 14 days after grafting with HA (a) or ZnHA (b and c). Asterisks indicate Multinucleated Giant Cells. (M) indicates the grafted area. (V) indicates blood vessels. The bar identifies a congested vessel. (CT) indicates connective tissue.

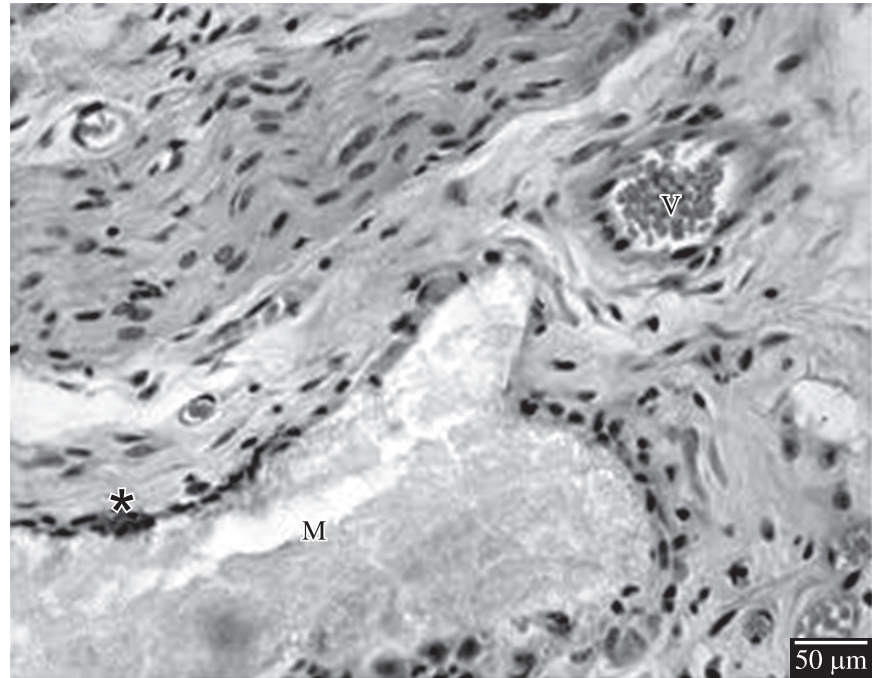

(a)

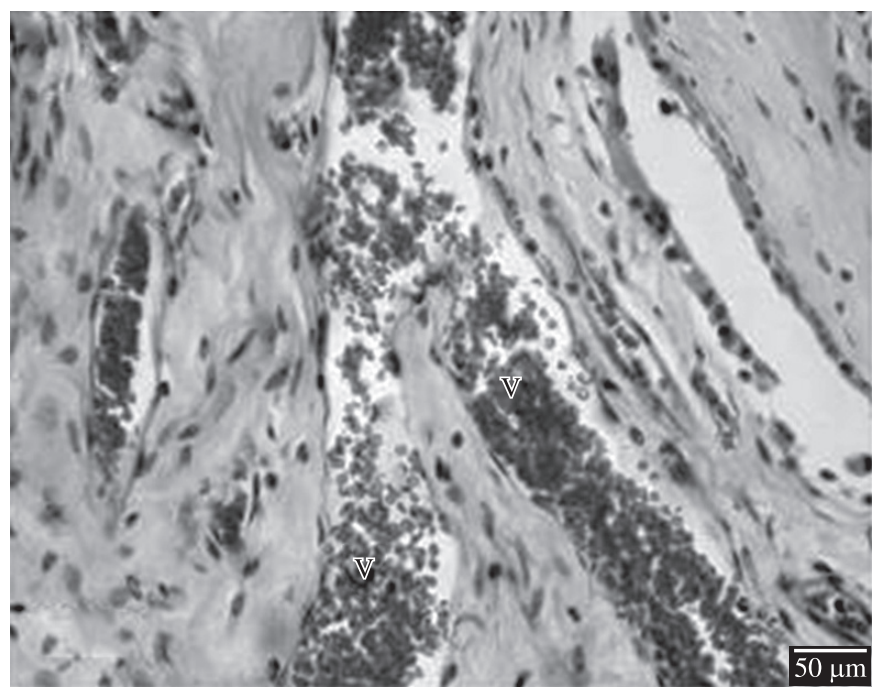

(b)

Figure 3. Histological sections, stained with HE, 28 days after grafting with HA (a) or ZnHA (b). Asterisks indicate Multinucleated Giant Cells. (M) indicates the grafted area. (V) indicates blood vessels. 


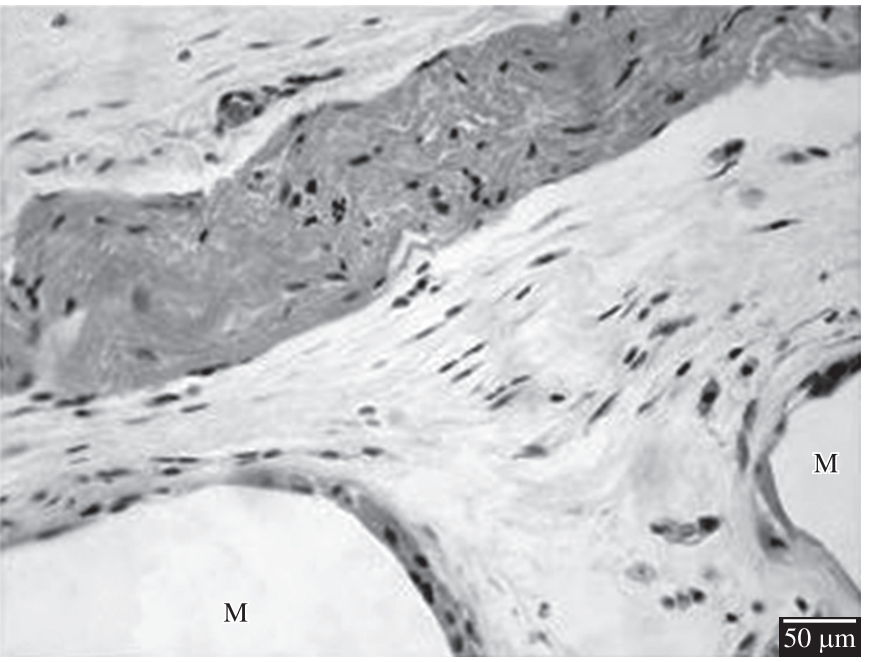

(a)

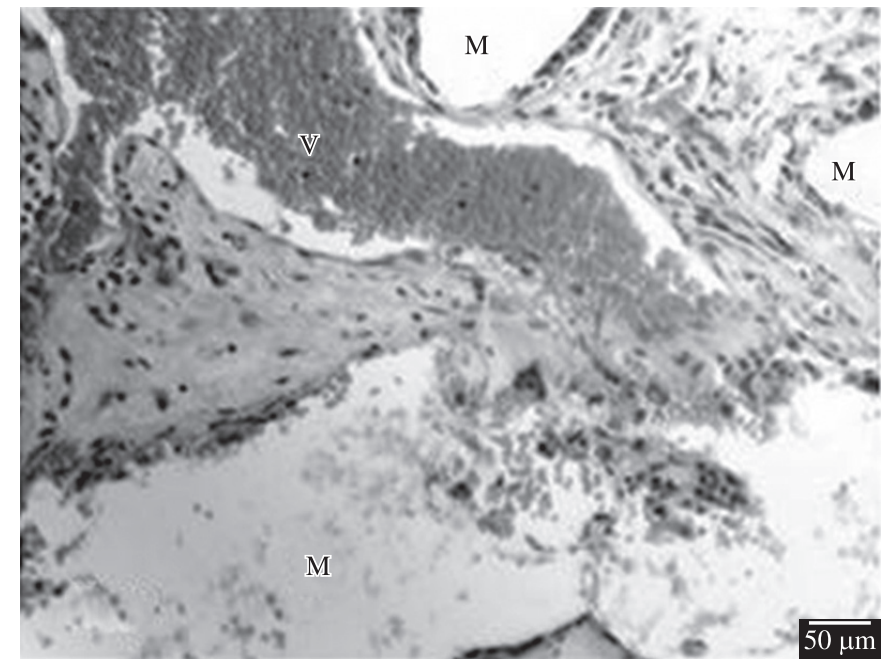

(b)

Figure 4. Histological sections, stained with HE, 60 days after grafting with HA (a) or ZnHA (b). (M) indicates the grafted area. (V) indicates blood vessels.

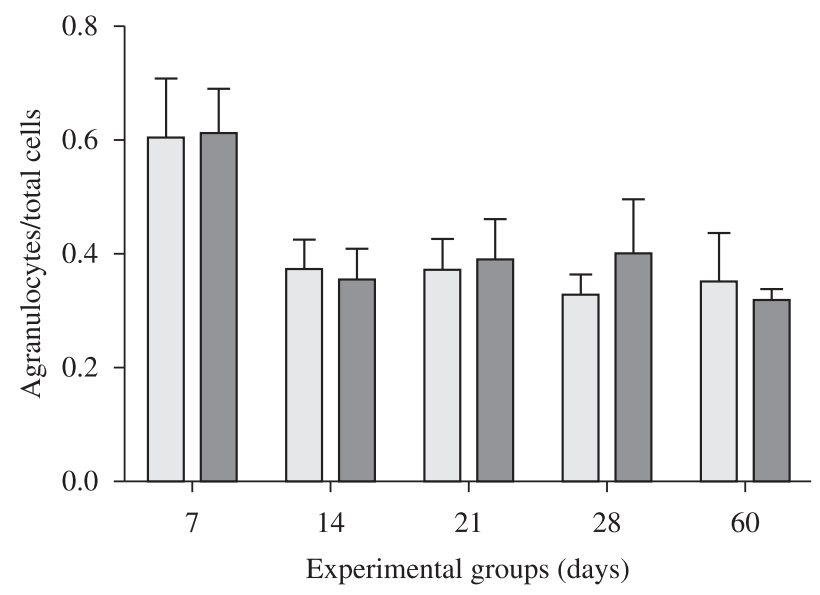

(a)

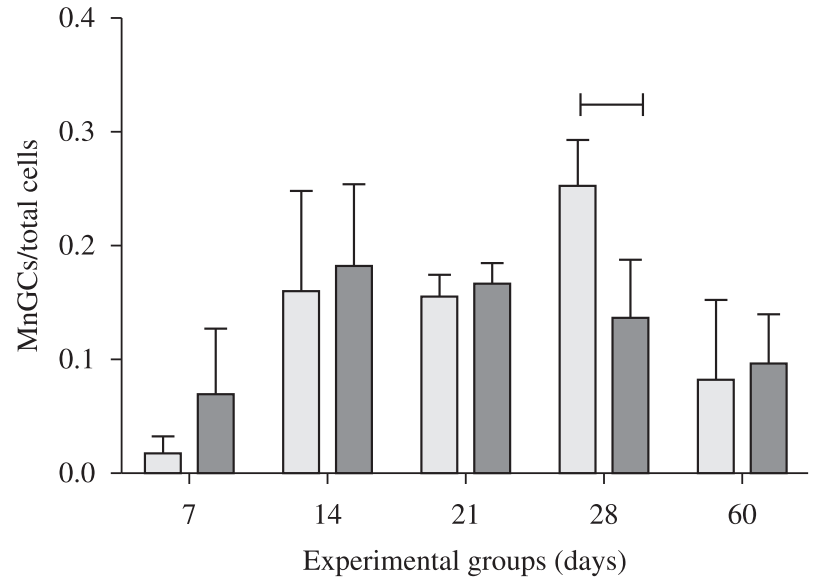

(c)

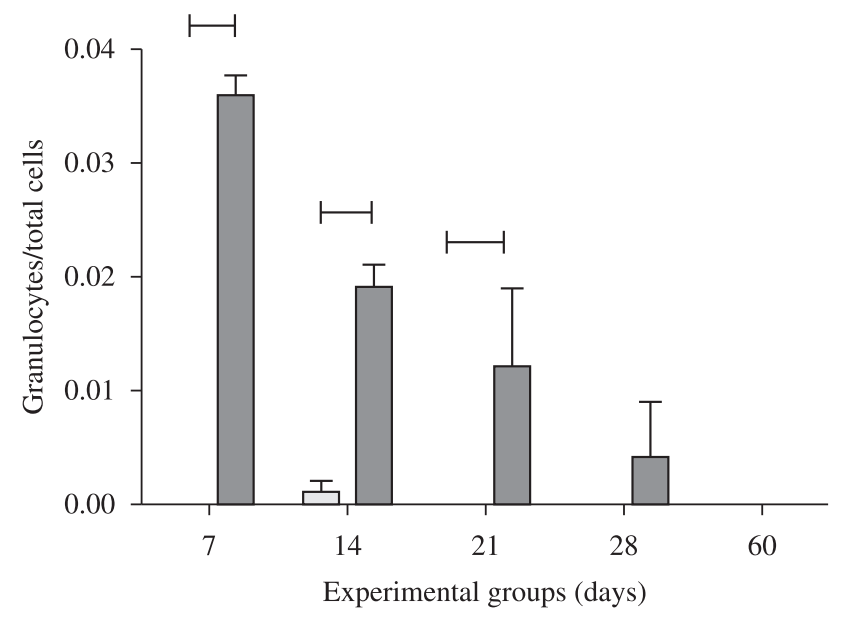

(b)

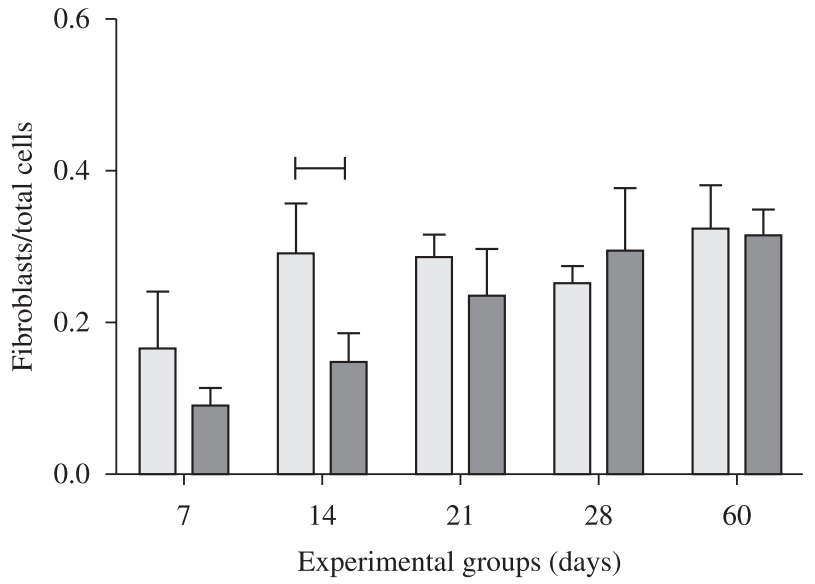

(d)

$\square$ HA $\square$ ZnHA

Figure 5. Histomorphometrical analysis of cell ditributions on the grafted areas. a) Agranulocytes; b) Granulocytes; c) Multinucleated Giant Cells (MnGCs); and d) Fibrocytes. Results represented as mean proportions of Total observed cells. Bars represent significant differences between groups ( $<<0.05$ ). 
during time of grafting, since samples from day 7 presented mean agranulocyte counting about two times higher $(\mathrm{p}<0.05)$ than those from subsequent experimental times (Figure 5a). Also, no significant difference was observed between $\mathrm{ZnHA}$ and stoichiometric HA on the estimated number of agranulocytes, on all evaluated periods.

When a biomaterial is implanted subcutaneously on an animal, the primary reaction of its nonspecific immune system is known as the Foreign Body Reaction (FBR), which shares several aspects of wound healing ${ }^{20}$. It is characterized by a specific molecular and cell dynamics, with the release of cytokines and leukocyte extravasation. The results hereby presented show that both tested materials induced a FBR biological response, with a granulomatous character, the presence of a fibrous capsule, and both acute and chronic processes characteristic of biocompatible grafted biomaterials. Agranulocytes represented the majority of cells detected, even tough their levels significantly decreased with time, suggesting the set of a mild chronic response accompanying the absorption of the material - a rather common response to biocompatible materials, usually due to the reduction of release of chemokines, eventually halting the influx of immune cells ${ }^{21}$.

The estimative of granulocytes for the ZnHA group was about 10 times lower than the number of agranulocytes $(\mathrm{p}<0.05)$ by day 7 , and decreased with time to rather irrelevant levels (Figure 5b). For the stoichiometric HA group, the presence of granulocytes was negligible, regardless of the grafting time. The virtual absence of granulocytes and PMN cells after 7 days contribute to the idea that any stronger immune response to both materials was probably resolved before the end of the first week after grafting, and no secondary response was initiated by both $\mathrm{HA}$ and $\mathrm{ZnHA}$.

Concerning multinucleated giant cells (MnGCs), Figure 5c shows significant differences between HA and ZnHA groups. The amounts of MnGCs for the ZnHA group presented its peak by day 14, while HA group levels kept arising, attaining significantly higher levels of multinucleated cells estimated at day 28, as compared to ZnHA Group ( $\mathrm{p}<0.05)$. The increase on the number of MnGCs distributed around the grafted area on both groups, from the 7 to the $28^{\text {th }}$ day with significant reduction by the $60^{\text {th }}$ day, indicates an important aspect of FBR. These cells are produced by the fusion of macrophages, as a result of the stimulation of different cytokines released on the grafted area, or of several cycles of "frustrated phagocytosis" by individual macrophages over large material surfaces ${ }^{22}$. The number of MnGCs, during the Foreign Body Reaction, tends to reduce over longer periods, as the size of the foreign particles reduces with adsorption ${ }^{20,22}$. According to present results, the amount of such cells on ZnHA treated samples was already significantly reduced at shorter times (28 days) than on the HA group. This fact possibly relates to the higher degradability of ZnHA granules, confirmed by the radiographic analysis of sample demineralization, and reported previously ${ }^{14}$. Also, it was described elsewhere that monocytes, in the presence of Zinc-containing apatites, tend to release lower levels of cytokines involved on the formation of $\mathrm{MnGCs}^{23}$.

The analysis for fibroblasts (Figure 5d) presented a rapid increase on the fibroblast content during the first 14 days, with a higher content of this cell type on the HA group during this period. However, no statistical difference was observed between groups starting from day 21.

Another important aspect of biomaterial compatibility is its angiogenic potential. The histomorphometrical analysis for angiogenesis revealed a faster increase on the mean number of blood vessels on samples from the ZnHA group during the first 14 days, as compared to stoichiometric HA (Figure 6).

The level of vascularization attained on this experimental group remained constant on subsequent times. Figure 6 shows that, with a

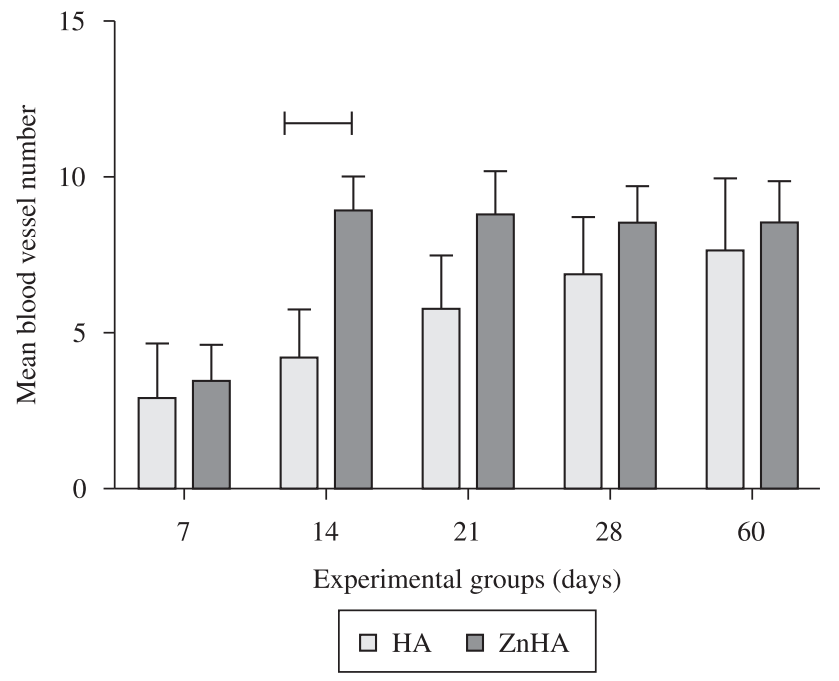

Figure 6. Histomorphometrical analysis of angiogenesis on the grafted areas. Results represented as mean counts of blood vessels. Bars represent significant differences between groups $(\mathrm{p}<0.05)$.

slower time-dependent increase on vascularization, only by day 60 the stoichiometric HA group approached the mean levels already attained for $\mathrm{ZnHA}$. In this manner, it seems that $\mathrm{ZnHA}$ is able to induce the formation of high-caliber vessels, mostly around the grafted area, on levels significantly higher than on HA samples, form 14 to 21 days. It is possible that zinc released by ZnHA may act on angiogenesis both by the stimulation of MMPs or zinc-finger proteins involved on VEGF gene activation ${ }^{24}$. Actually, zinc release from the biomaterial to biological media is a rather expected phenomenon, on the light of the high solubility of such granules under several different conditions, as described previously ${ }^{14}$.

However, other possible positive biological effects of zinc, such as the induction of bone tissue differentiation ${ }^{9}$ were not observed in this work. In this regard, it is important to note that the animal model employed here - subcutaneous grafting on mice - even tough ideal for biocompatibility tests, is not favorable to the observation of osteoinduction on heterotropic tissue, which would be more adequately observed with intramuscular grafting ${ }^{25}$.

\section{Conclusions}

Based on the obtained results, it could be concluded that $\mathrm{ZnHA}$ granules are at least as biocompatible as non-modified HA, since both performed very similarly on a standard ectopic in vivo assay. Moreover, our data indicate both an improved angiogenesis and a faster reduction on the levels of cells involved on inflammatory response in the presence of $\mathrm{Zn}-\mathrm{HA}$, suggesting that the incorporation of Zinc might improve the performance of hydroxyapatite. Further studies should better address its potential use for bone therapy, mainly concerning the analysis of other zinc concentration to incorporate to $\mathrm{HA}$ and more detailed analysis to understand the relation of $\mathrm{ZnHA}$ and angiogenesis.

\section{Acknowledgements}

Authors acknowledge the financial support from CAPES, CNPq and FAPERJ, and the technical support from Dr. Alexandre Rossi, at CBPF, for the production of hydroxyapatite powders, as well as the Núcleo de Animais de Laboratório from the Fluminense Federal University (NAL-UFF), for the maintenance of the animals used on this study. 


\section{References}

1. El-Ghannam A. Bone reconstruction: from bioceramics to tissue engineering. Expert Review on Medical Devices. 2005; 2:87-101.

2. Dorozhkin SV. Bioceramics of calcium orthophosphates. Biomaterials. 2010; 31:1465-1485.

3. Porter JR, Ruckh TT and Popat KC. Bone tissue engineering: a review in bone biomimetics and drug delivery strategies. Biotechnology Progress. 2009; 25:1539-1560.

4. Fadeev IV, Shvorneva LI, Barinov SM and Orlovskii VP. Synthesis and structure of magnesium-substituted hydroxyapatite. Inorganic Materials. 2003; 39:947-950.

5. Landi E, Logroscino G, Proietti L, Tampieri A, Sandri M and Sprio S. Biomimetic Mg-substituted hydroxyapatite: from synthesis to in vivo behaviour. Journal of Material Sciences: Materials in Medicine. 2008;19:239-247.

6. Mehnaoui M, Panczer G, Ternane R, Trabelsi-Ayedi M and Boulon G. Structural and spectroscopic characterizations in $\mathrm{Pb} 2+$-doped calcium hydroxyapatites. Optical Materials. 2008; 30:1672-1676.

7. Ni GX, Lu WW, Chiu KY, Li ZY, Fong DY and Luk KD. Strontiumcontaining hydroxyapatite (Sr-HA) bioactive cement for primary hip replacement: an in vivo study. Journal of Biomedical Materials Research part B Applied Biomaterials. 2006; 77:409-415.

8. Wu X, Itoh N, Taniguchi T, Nakanishi T, Tatsu Y, Yumoto N et al. Zinc-induced sodium-dependent vitamin $\mathrm{C}$ transporter 2 expression: potent roles in osteoblast differentiation. Archives on Biochemistry and Biophysics. 2003; 420:114-120.

9. Storrie $\mathrm{H}$ and Stupp SI. Cellular response to zinc-containing organoapatite: an in vitro study of proliferation, alkaline phosphatase activity and biomineralization. Biomaterials. 2005; 26:5492-5499.

10. Relea P, Revilla M, Ripoll E, Arribas I, Villa LF and Rico H. Zinc, biochemical markers of nutrition, and type I osteoporosis. Age \& Ageing. 1995; 24:303-307.

11. Fernandes GVO, Calazans-Maia MD, Mitri FF, Rossi AM, Almeida GDS and Granjeiro JM. Histomorphometric Analysis of Bone Repair in Critical Size Defect in rats Calvaria Treated with Hydroxyapatite and Zinc-Containing Hydroxyapatite 5\%. Key Engineering Materials. 2009; 396:15-18.

12. Ito A, Otsuka M, Kawamura H, Ikeuchi M, Ohgushi H, Sogo Y et al. Zinc-containing tricalcium phosphate and related materials for promoting bone formation. Current Applied Physics. 2005; 5:402-406.
13. Kawamura H, Ito A, Muramatsu T, Miyakawa S, Ochiai N and Tateishi T. Long-term implantation of zinc-releasing calcium phosphate ceramics in rabbit femora. Journal of Biomedical Materials Research Part A. 2003; 65A:468-474

14. Lima IR, Costa AM, Bastos IN, Granjeiro JM and Soares GA. Development and Characterization of $5 \%$ mol Zn Bioceramic in Granular Form. Materials Research. 2006; 9:399-403.

15. Otsuka M, Marunaka S, Matsuda Y, Ito A, Layrolle $\mathrm{P}$, Naito $\mathrm{H}$ et al. Calcium level-responsive in-vitro zinc release from zinc containing tricalcium phosphate (ZnTCP). Journal of Biomedical Materials Research. 2000; 52:819-824.

16. Du RL, Chang J, Ni SY, Zhai WY and Wang JY. Characterization and in vitro bioactivity of zinc-containing bioactive glass and glass-ceramics. Journal of Biomaterial Applications. 2006; 20:341-360.

17. Costa AM, Soares GA, Calixto R and Rossi AM. Preparation and properties of zinc containing biphasic calcium phosphate bioceramics. Key Engineering Materials. 2004; 254-256:119-122.

18. ASTM. ASTM F748 - 06 (2010) Standard Practice for Selecting Generic Biological Test Methods for Materials and Devices; 2010.

19. International Organization for Standardization. ISO 10933-6:2007 Biological evaluation of medical devices -- Part 6: Tests for local effects after implantation; 2007.

20. Luttikhuizen DT, Harmsen MC and van Luyn MJ. Cellular and molecular dynamics in the foreign body reaction. Tissue Engineering. 2006; 12:1955-1970.

21. Gillitzer R and Goebeler M. Chemokines in cutaneous wound healing. Journal of Leukocyte Biology. 2001; 69:513-521.

22. Anderson JM, Rodriguez A and Chang DT. Foreign body reaction to biomaterials. Seminars in Immunology. 2008; 20:86-100.

23. Grandjean-Laquerriere A, Laquerriere P, Jallot E, Nedelec JM, Guenounou M, Laurent-Maquin D et al. Influence of the zinc concentration of sol-gel derived zinc substituted hydroxyapatite on cytokine production by human monocytes in vitro. Biomaterials. 2006; 27:3195-3200.

24. Pasqualini R and Barbas CF, III and Arap W. Vessel maneuvers: zinc fingers promote angiogenesis. Nature Medicine. 2002; 8:1353-1354.

25. Habibovic P, Sees TM, van den Doel MA, van Blitterswijk CA, de Groot $\mathrm{K}$. Osteoinduction by biomaterials--physicochemical and structural influences. Journal of Biomedical Materials Research Part A. 2006; 77:747-762. 